\title{
GEORobótica - Uma proposta lúdica interdisciplinar para Ensino de Geografia no Ensino Médio: um relato de experiência da robótica educacional com alunos de escola pública
}

\author{
Fernando Lucas de Oliveira Farias, Everton da Silva Brito, Felipe Jhonanta Ferreira da \\ Costa, Igo Joventino Dantas Diniz, Akynara Aglaé Rodrigues Santos da Silva Burlamaqui \\ e Aquiles Medeiros Filgueira Burlamaqui \\ Instituto Metrópole Digital - Universidade Federal do Rio Grande do Norte (UFRN) \\ Av. Sen. Salgado Filho, 3000 - Lagoa Nova, CEP: 59.078-970 - Natal - RN - Brasil \\ \{fernandoo.mcp, evertondsb36, felipejfc,igojoven, akynara, \\ aquilesburlamaqui\} egmail.com
}

\begin{abstract}
The promotion of interdisciplinary educational practices based on transversal themes supported by educational technologies has been one of the great challenges faced in K-12. In the discipline of Geography this reality is not different and even more challenging. In this sense, the work aims to present an experience report with students of a public school participating in a playful and interdisciplinary proposal for teaching geography with educational robotics entitled "GEORobótica". The methodology adopted in the experiment was supported by Design Thinking and PBL. The results of the research indicate that the educational practice was considered playful, challenging and able to contextualize interdisciplinarily concepts of geography and robotics.
\end{abstract}

Resumo. A promoção de práticas educativas interdisciplinares pautadas em temas transversais apoiadas por tecnologias educacionais, tem sido um dos grandes desafios enfrentados na educação básica. Na disciplina de Geografia essa realidade não é diferente e ainda mais desafiadora. Neste sentido, o trabalho tem como objetivo apresentar um relato de experiência com alunos do ensino médio de uma escola pública participantes de uma proposta lúdica e interdisciplinar para ensino de geografia com robótica educacional intitulada "GEORobótica". A metodologia adotada no experimento foi apoiada pelo Design Thinking e o PBL. Os resultados da pesquisa apontam que a prática educativa foi considerada lúdica, desafiadora e capaz de contextualizar interdisciplinarmente conceitos da geografia e a robótica.

\section{Introdução}

A robótica como postura pedagógica interdisciplinar em disciplinas da educação básica tem sido uma abordagem pouco explorada na literatura, principalmente em cenários educacionais da rede pública, seja no ensino fundamental ou médio.

O ensino de Geografia tem como papel promover nos estudantes uma reflexão crítica acerca da compreensão do mundo em que vivem, complexidades do mundo contemporâneo e as interações da sociedade e natureza; caracterizando-se epistemologicamente pela busca de respostas para questões relacionadas ao tempo, ao espaço e ao lugar [Bomfim 2006]. 
A robótica na escola pode ser assimilada como práticas educativas que envolvam atividades de construção, manipulação e programação de robôs, estimulando a construção de habilidades ligadas ao pensamento computacional, raciocínio lógico, pensamento crítico, trabalho em equipe numa perspectiva integrada e transversal [Peralta e Guimarães 2018].

As metodologias ativas de ensino dão ênfase ao papel protagonista do aprendiz, ao seu envolvimento direto, participativo e reflexivo em todas as etapas do processo, experimentando, desenhando, criando, com a orientação do professor utilizadas na estruturação metodológica e sequenciamento didático da prática educacional desenvolvida [Bacich; Moran 2017]

Neste contexto, a intervenção didático-pedagógica apresentada neste relato foi realizada com estudantes de uma escola pública estadual do Rio Grande do Norte - RN, onde foram estabelecidas relações entre a Geografia e a robótica; a fim de promover uma compreensão interdisciplinar da Revolução Técnico-científica-informacional e os impactos no mercado global.

Em nosso experimento, apresentamos na fase de imersão inicial com os alunos, conceitos sobre robótica educacional e seus componentes, potencialidades da robótica frente a construção das habilidades do profissional do futuro ${ }^{1}$ propostas pelo Fórum Econômico Mundial, possibilitando a compreensão do papel da tecnologia como agente propulsor da globalização.

Em análise preliminar através de uma pesquisa semi-estruturada ${ }^{2}$ realizada com os participantes ao final do experimento, verificamos que $85,7 \%$ não haviam participado anteriormente de aulas interdisciplinares com o uso da robótica educacional; sendo que 90,5\% julgam muito importante a inserção da proposta em outras disciplinas, reiterando a percepção inicial dos pesquisadores que a robótica educacional ainda é pouco explorada em práticas educativas nas escolas públicas de ensino no Brasil.

Por conseguinte, a proposta lúdica interdisciplinar para ensino de Geografia no Ensino Médio aqui intitulada "GEORobótica", é apresentada como referência de cenário educacional integrando robótica e a Geografia para apresentar a inter-relação entre os conceitos de robótica, a dinâmica de produção mundial e o mercado de trabalho para estudantes da educação básica.

O trabalho está organizado da seguinte forma: na Seção 2 são apresentados trabalhos relacionados a utilização da robótica educacional no ensino médio como proposta lúdica interdisciplinar; a Seção 3 relaciona os objetivos da prática educativa desenvolvida; a Seção 4 apresenta a metodologia utilizada no desenvolvimento do trabalho; a Seção 5 apresenta os resultados obtidos durante o experimento e discussão sobre o engajamento dos estudantes, por fim, a Seção 6 detalha as Considerações Finais e apresenta possibilidades para Trabalhos Futuros.

\section{Trabalhos Relacionados}

Dentre os trabalhos relacionados, podemos citar a pesquisa de Sousa (2017) que utiliza kits de robótica como recurso tecnológico nas aulas de Geografia para apresentação e demonstração das fontes de energia renováveis. Os resultados da montagem do robô movido à energia solar demonstram acentuado engajamento dos estudantes e o forte trabalho em equipe, identificado

\footnotetext{
${ }^{1}$ Disponível em: $<$ http://www3.weforum.org/docs/WEF_Future_of_Jobs.pdf $>$ Acesso em: 17 out. 2018

2 Disponível em: $<$ http://bit.ly/berilo-georobótica $>$ Acesso em: 17 out. 2018
} 
através do empenho coletivo na montagem do kit, tornando as aulas mais dinâmicas e interativas; possibilitando na percepção dos autores a construção inovadora de conceitos geográficos.

Benitti et al. (2009), apresenta um experimento, denominado "Viajando por Santa Catarina" que permitiu aos alunos realizarem atividades de robótica aplicando conceitos relacionados à matemática, geografia e programação de computadores, especificamente pontos cardeais, plano cartesiano, mapa de Santa Catarina e lógica de programação. Os resultados foram considerados promissores pelo engajamento dos estudantes em realizar outras atividades no "RoboLAB: Ambiente de experimentação científico-tecnológico em robótica educativa".

Puziski et al. (2018), descreve uma proposta interdisciplinar com alunos de uma turma de quinto ano do ensino fundamental envolvendo as disciplinas de geografia, ciências, língua portuguesa e robótica. A proposta consiste em atividades envolvendo modelo robótico que simula os movimentos da Terra e da Lua, intitulado "Terra-Lua-Sol", os resultados apresentados indicam que a atividade interdisciplinar proporcionou aos estudantes o desenvolvimento do conhecimento e habilidades previstas nos objetivos de aprendizagem propostos.

Neste sentido, a proposta intitulada GEORobótica distingue-se dos trabalhos relacionados por explorar um cenário educacional planejado cuidadosamente para contextualização dos objetivos de aprendizagem relacionados à terceira revolução industrial com a utilização mínima de recursos e/ou infraestrutura, especificamente trabalhamos em nossa intervenção com um Kit Lego Mindstorm v3 ${ }^{3}$ que foi montado colaborativamente em quatro etapas, coordenadas sistematicamente por quatro grupos compostos pelos vinte e oito alunos da turma.

\section{Objetivos}

Os objetivos da proposta levaram em consideração tangencialmente algumas habilidades relacionadas ao ensino de Computação para as etapas da Educação Básica propostas pela Sociedade Brasileira de Computação - SBC através do documento ${ }^{4}$ que trata dos referenciais de formação em computação para a Educação Básica, dentre elas podemos destacar no eixo Pensamento Computacional (PC) "capacidade de sistematizar, representar, analisar e resolver problemas, considerado um dos pilares fundamentais do intelecto humano; serve para descrever, explicar e modelar o universo e seus processos complexos" [SBC 2017].

\subsection{Objetivo geral}

Apresentar a inter-relação entre os conceitos de robótica, a dinâmica de produção mundial e o mercado de trabalho.

\subsection{Objetivos específicos}

- Entender a relação entre robótica e a educação;

- Identificar as novas habilidades necessárias ao profissional do futuro;

- Compreender o papel da tecnologia como agente propulsor da globalização;

- Analisar a espacialização e fragmentação do processo de produção no contexto globalizado.

\footnotetext{
3 Disponível em: <https://www.lego.com/pt-br/mindstorms/about-ev3> Acesso em: 17 out. 2018

4 Disponível em: $<$ http://bit.ly/2LMcevX> Acesso em: 17 out. 2018
} 
VIII Congresso Brasileiro de Informática na Educação (CBIE 2019)

Anais do XXV Workshop de Informática na Escola (WIE 2019)

\section{Metodologia}

A realização da prática educativa seguiu um plano de aula ${ }^{5}$ elaborado colaborativamente por uma equipe multidisciplinar constituída por profissionais das áreas de computação, administração, design gráfico e geografia que foram responsáveis pela definição da temática da intervenção, objetivos, conteúdos, sequenciamento didático, critérios de avaliação e resultados esperados.

O cenário educacional teve início com processo de imersão inicial apoiado pelo Design Thinking, onde os estudantes foram apresentados aos conceitos (i) robótica X mercado de trabalho; (ii) robótica X 10 top habilidades do profissional do futuro; (iii) robótica $\mathrm{X}$ automação de processos (indústria 4.0); (iv) revisão sobre a terceira revolução industrial através da exibição de um vídeo ${ }^{6}$ e (v) apresentação da cadeia produtiva de veículos em âmbito global.

Segundo Vianna et al. (2012), o Design Thinking é caracterizado como sendo um processo centrado no entendimento da problemática de forma aprofundada para só então se propor soluções que a resolva de forma efetiva, sendo alcançado por meio de uma imersão profunda no contexto do problema, buscando as verdadeiras raízes da questão investigada.

A segunda fase do experimento foi iniciada com apresentação de uma situação-problema onde os estudantes deveriam simular uma linha de produção/montagem à luz da cadeia produtiva de veículos para montagem de um Kit Lego Mindstorm v3 denominado de "RobôGEO", sendo o processo apoiado pela metodologia do Problem Based Learning (PBL).

O Kit educacional da LEGO $^{\circledR}$, o Mindstorms, possui centenas de componentes para montagem de robôs incluindo a estrutura do robô, sensores e atuadores que possibilitam montar um portfólio variado de robôs, estimulando a criatividade na resolução de problemas; bem como disponibiliza uma interface de programação visual baseada em blocos coloridos que possibilitam a formação de sequência de ações que em seguida são enviadas ao robô para realização das mais diversas atividades [De Oliveira et al. 2013; Aguiar et al. 2015]

Segundo Monteiro et al. (2011), "a aprendizagem baseada em problemas estimula e favorece o trabalho em equipe, melhor a assimilação de conteúdos e aumenta a motivação para aprender”, o que foi identificado nos estudantes participantes da intervenção durante as interações para montagem do RobôGEO.

Savery (2015), esclarece que a metodologia baseada em problemas deve "capacitar os estudantes a realizar pesquisas, integrar a teoria e prática e aplicar conhecimentos e habilidades para desenvolver uma solução viável para um problema definido”.

Na Tabela 1 são descritas as quatro etapas de montagem do RobôGEO realizadas pelos estudantes da Escola $\mathrm{X}$ do estado $\mathrm{Y}$, reunidos em equipes com até sete membros; realizada num tempo estimado de quarenta e cinco a sessenta minutos, ilustradas nas Figuras 1, 2, 3 e 4.

\footnotetext{
5 Disponível em: <http://bit.ly/2MRCcL6> Acesso em: 17 out. 2018

${ }^{6}$ Disponível em: $<$ https://www.youtube.com/watch?v=rr5LZ32M_s $>$ Acesso em: 17 out. 2018
} 
VIII Congresso Brasileiro de Informática na Educação (CBIE 2019)

Anais do XXV Workshop de Informática na Escola (WIE 2019)

Tabela 1. Etapas de Montagem do RobôGEO

\begin{tabular}{|c|l|c|}
\hline Etapa & \multicolumn{1}{|c|}{ Descrição } & Tempo Estimado \\
\hline Etapa 1 & $\begin{array}{l}\text { Montagem dos componentes "The Vehicle" seguindo } \\
\text { às orientações das páginas 2 a 35 do manual7 } \\
\text { disponibilizado pela LEGO® }\end{array}$ & 20min \\
\hline Etapa 2 & $\begin{array}{l}\text { Montagem de sensores para acoplamento no "The } \\
\text { Vehicle" seguindo às orientações das páginas 55 a 60 } \\
\text { e 69 a 76 do manual disponibilizado pela LEGO® }\end{array}$ & 10min \\
\hline Etapa 3 & $\begin{array}{l}\text { Montagem do Microcontrolador para acoplamento do } \\
\text { "The Vehicle" seguindo às orientações das páginas 36 } \\
\text { a 46 do manual disponibilizado pela LEGO® }\end{array}$ & 5min \\
\hline Etapa 4 & $\begin{array}{l}\text { Montagem do motor médio para acoplamento no "The } \\
\text { Vehicle" seguindo às orientações das páginas 78 a 97 }\end{array}$ & 10min \\
\hline
\end{tabular}

Fonte: Elaborado pelos autores

Figura 1: Etapas 1 e 2 de Montagem do RobôGEO
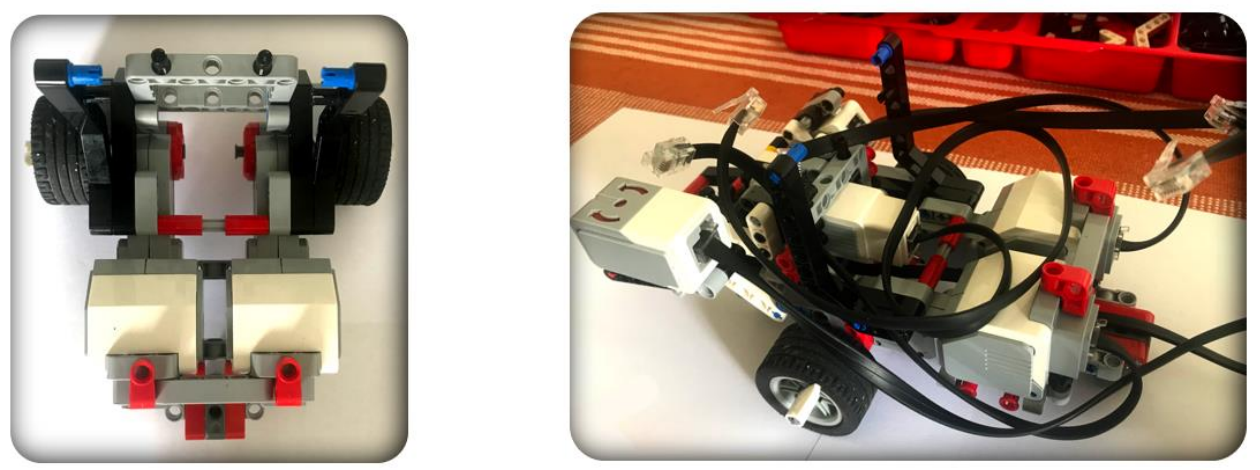

Fonte: Elaborado pelos autores

Figura 2: Etapas 3 e 4 de Montagem do RobôGEO
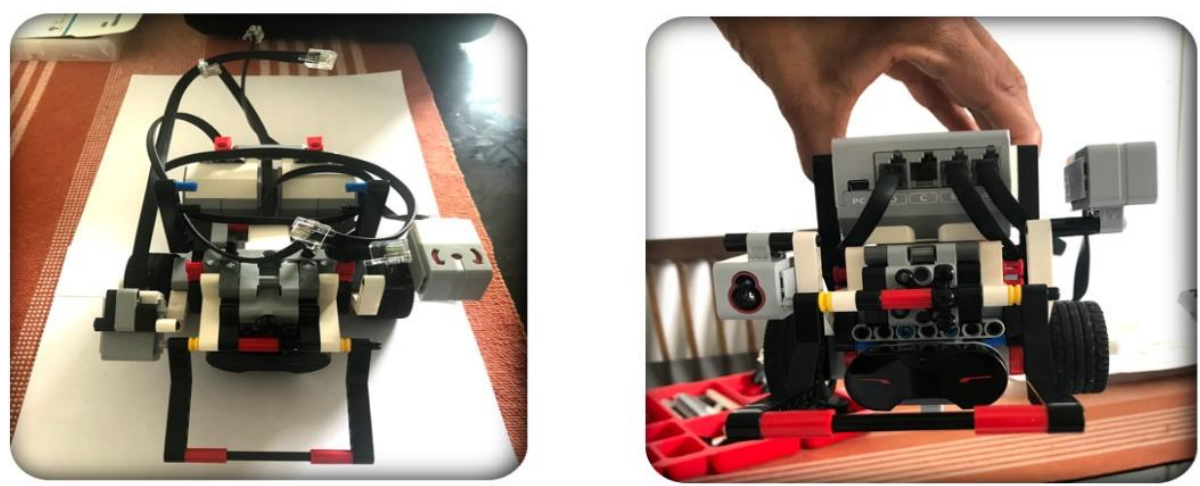

Fonte: Elaborado pelos autores

\footnotetext{
7 Disponível em: $<$ http://bit.ly/2QYNsYK > Acesso em: 18 out. 2018

${ }^{8}$ Disponível em: <http://bit.ly/2QYNsYK> Acesso em: 18 out. 2018
} 
A fim de manter os alunos engajados em suas estações de aprendizagem no curso da montagem do RobôGEO e proporcionar consolidação da imersão inicial, foi solicitado às equipes a produção colaborativa de um mapa mental dentro das seguintes temáticas: (i) Quais os países que mais fornecem matéria prima para produção de veículo; (ii) Quais os países mais produtores de veículos; (iii) Quais os países que mais exportam os veículos e (iv) Quais países que mais compram veículos, sendo que cada equipe foi encarregada de ilustrar em cartolina disponibilizada no experimento, os dados de sua temática por meio da modelagem de um mapa mental.

Segundo Marques (2008), o mapa mental é uma ferramenta que permite a estruturação de informação de forma não linear, enfatizando a ideia principal no centro e as ideias associadas descritas com palavras-chave que podem ser ilustradas com imagens, ícones e cores variadas

Por conseguinte, após montagem do RobôGEO cada equipe teve que operar o robô para os países onde foram identificadas a maior produção de matéria-prima, produção de veículos, exportação de veículos e mercado consumidor divulgados, fruto das pesquisas realizadas.

Figura 3: Aluno operando o RobôGEO após sua montagem colaborativa

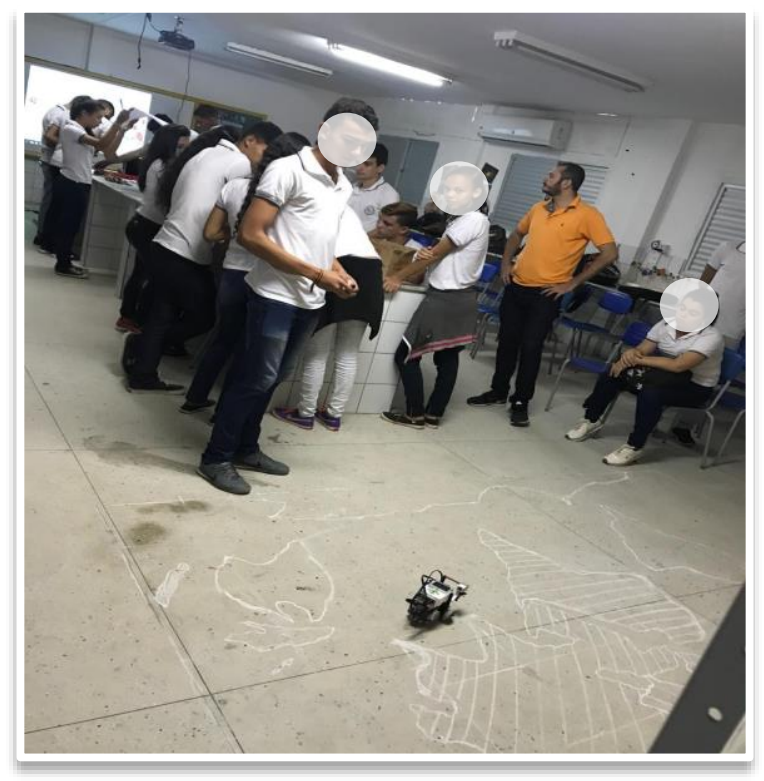

Fonte: Elaborado pelos autores

Ao final do experimento foi realizada uma avaliação de reação através de um survey ${ }^{9}$ que teve como objetivo principal colher informações, acerca da percepção dos estudantes sobre a experiência denominada "GEORobótica", enquanto proposta lúdica e interdisciplinar para ensino de geografia no ensino médio apoiada na robótica educacional.

\section{Resultados e Discussões}

Nesta seção, iremos discutir os dados coletados durante a avaliação de reação realizada com os estudantes participantes da aula estruturada com a proposta "GEORobótica", incluindo sua percepção quanto ao caráter lúdico da abordagem, importância da inserção da robótica em outras disciplinas, habilidades desenvolvidas, aspectos positivos e negativos identificados na proposta.

${ }^{9}$ Disponível em: $<$ http://bit.ly/berilo-georobótica $>$ Acesso em: 18 out. 2018 
VIII Congresso Brasileiro de Informática na Educação (CBIE 2019)

Anais do XXV Workshop de Informática na Escola (WIE 2019)

Na Figura 4 é ilustrada a participação anterior dos estudantes em aulas interdisciplinares com uso da robótica educacional, identificamos que 85,7\% não haviam vivenciado tal experiência. Neste sentido, a experiência anterior relatada por $14,3 \%$ dos estudantes foi desenvolvida em outras instituições de ensino, sendo a intervenção realizada o primeiro contato da maioria com a robótica.

Figura 4: Participação anterior em aulas interdisciplinares com uso da robótica educacional

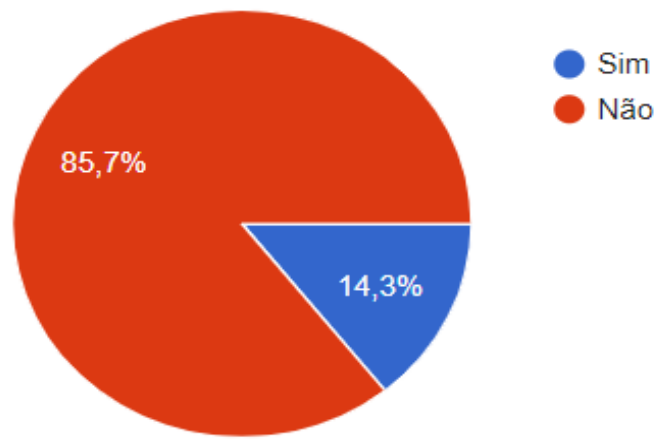

Fonte: Elaborado pelos autores

O instrumento buscou mensurar o nível de diversão proporcionado pela atividade numa escala de 1 - Pouco divertida a 5 - Muito divertida na percepção dos estudantes, bem como compreensão do nível de importância quanto a inserção da robótica educacional em outras disciplinas numa escala de 1 - Pouco importante e 5 - Muito importantes, ver Figuras 5 e 6.

Figura 5. Avaliação pelos alunos do nível de diversão proporcionado pela atividade GEORobótica

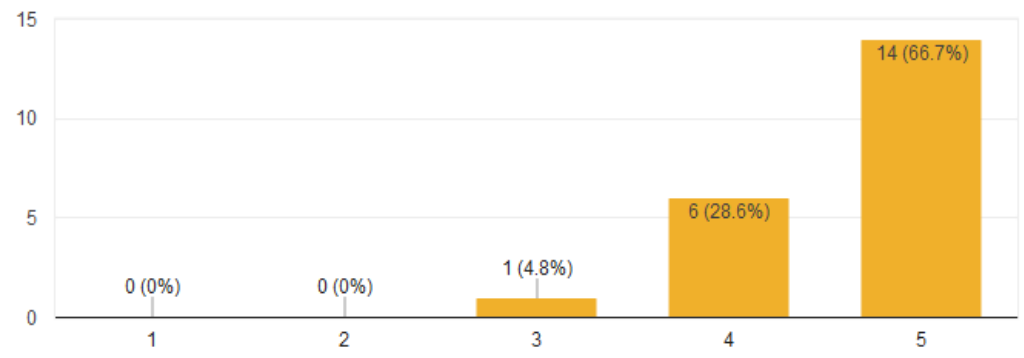

Fonte: Elaborado pelos autores

Figura 6: Avaliação do nível de importância da inserção da robótica em outras disciplinas

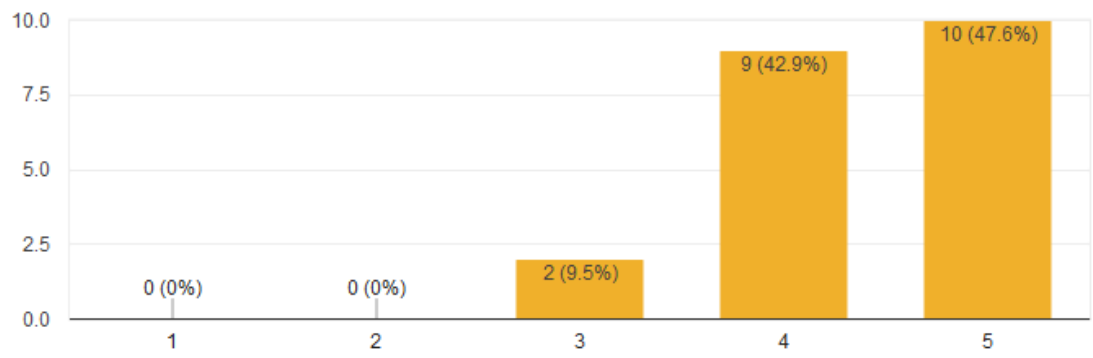

Fonte: Elaborado pelos autores 
Os dados das Figuras 5 e 6 reforçam a percepção dos pesquisadores mediadores da prática educativa quanto a satisfação e engajamento dos estudantes na montagem do RobôGEO e construção do mapa mental durante a intervenção, principalmente pelo coeso trabalho em equipe desenvolvido pelos aprendizes no curso das atividades, ilustrado nas Figuras 7 e 8.

Figura 7: Montagem do RobôGEO pela equipe 1

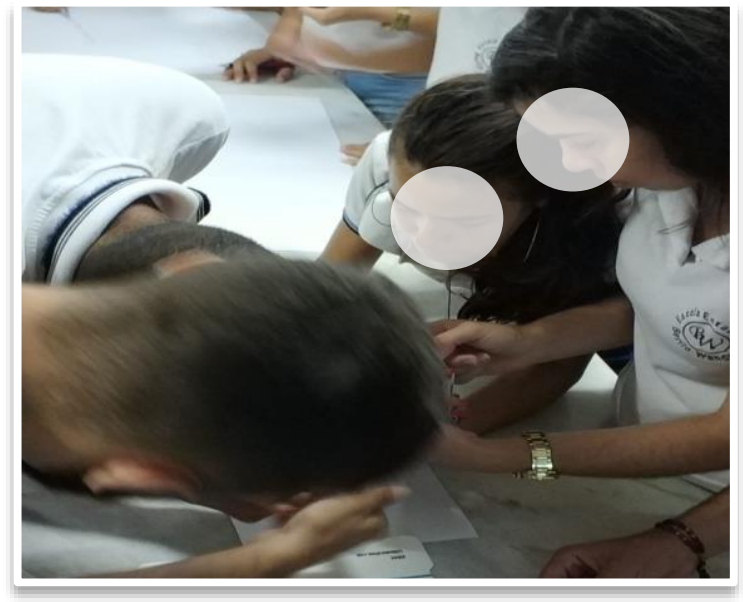

Figura 8: Montagem do RobôGEO pela equipe 3

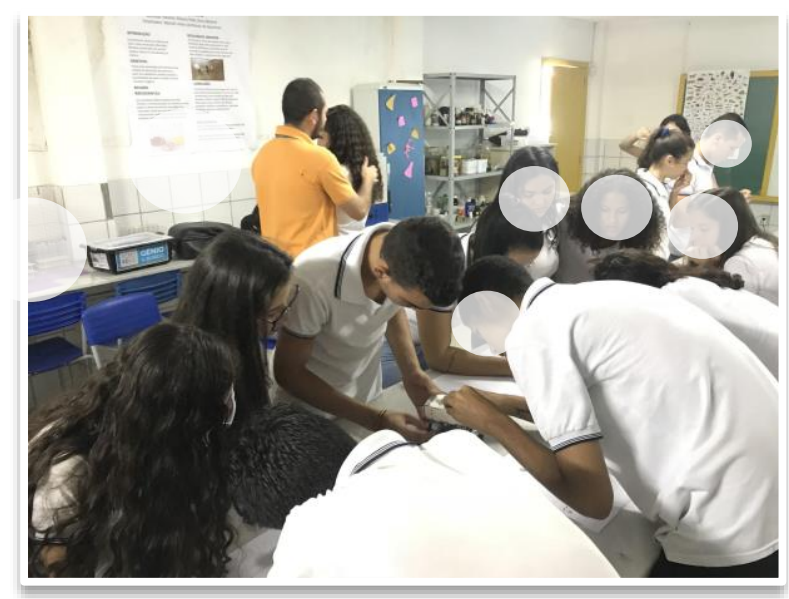

Figura 9: (a) Avaliação da melhor compreensão da inter-relação dos conteúdos; (b) Avaliação da melhor compreensão do papel da tecnologia como agente propulsor da globalização

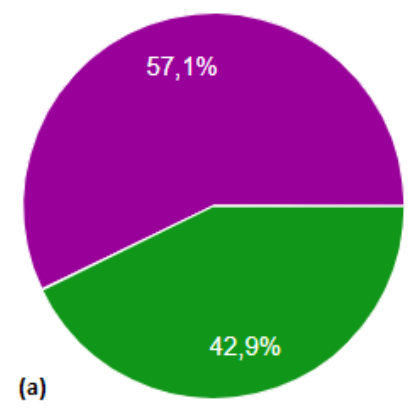

Discordo totalmente (DT)

Discordo parcialmente (DP)

Indiferente (I)

Concordo parcialmente (CP)

Concordo totalmente (CT)

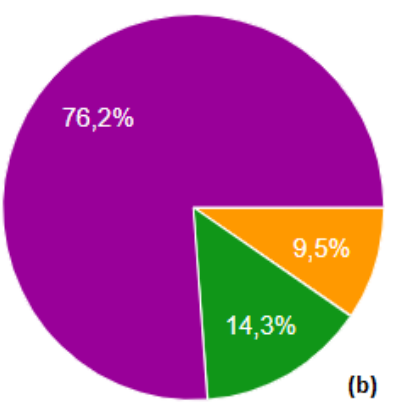

(b)

Fonte: Elaborado pelos autores

Os dados das Figuras 9a e 9b são valiosos e promissores, indicando boa aceitação da prática "GEORobótica" como proposta interdisciplinar exitosa ao facilitar a compreensão da inter-relação entre os conceitos de robótica, dinâmica de produção mundial e o mercado de trabalho na percepção de $100 \%$ dos participantes, ver Figura 9a, enquanto 90,5\% concordam que a prática educativa permitiu entender o papel da tecnologia como agente propulsor da globalização, ver Figura 9b. Tais dados refletem, os benefícios proporcionados pelas estratégias metodológicas utilizadas no desenvolvimento do estudo pautadas no Design Thinking e PBL.

Não obstante, procuramos avaliar a experiência de montagem do primeiro robô pelos estudantes, os feedbacks foram bastante positivos, dentre eles destacamos: "Momento proveitoso onde nós tivemos a sorte de ser a turma escolhida, experiência que posso levar para o mercado de 
trabalho"; "Legal, Interessante e Bem divertido"; "Um pouco complicado, mas montando com atenção sempre dá certo"; "Sugiro mais projetos como esse, não só na geografia, acho que inserir em outras matérias será interessante, do modo de cada professor"; "Muito, muito, muito interessante! Gostei de simplesmente tudo da aula. Desde os professores até a parte prática. Minha única crítica é o pouco tempo! Minha sugestão é que tenha mais aulas desse tipo em outras matérias e que seja muito bem aproveitado.”.

\section{Considerações finais}

A proposta intitulada "GEORobótica" apresentou-se como desafiadora desde sua concepção, alinhar objetivos de aprendizagem da geografia numa perspectiva interdisciplinar com a robótica, computação e a matemática não foi uma tarefa trivial, demandando bastante empenho, criatividade e resiliência dos pesquisadores envolvidos na intervenção.

Todavia, os resultados obtidos com a prática educativa foram bastante positivos, valiosos e promissores; principalmente pela forte aceitação da proposta por parte dos aprendizes que se mantiveram engajados durante todo o cenário educacional, atingindo os objetivos estabelecidos em nosso plano de aula, bem como obtendo resultados satisfatórios nos processos de avaliação estabelecidos.

A principal contribuição de nosso trabalho consiste em apresentar um cenário educacional onde com a utilização de apenas um kit de robótica educacional para uma turma de 28 alunos matriculados numa escola pública de ensino, foi possível o desenvolvimento de uma proposta interdisciplinar para ensino de geografia onde competências fundamentais a formação de profissionais do futuro foram trabalhadas: resolução de problemas complexos; pensamento crítico; inteligência emocional; bom senso e tomada de decisões; criatividade e empatia com os outros em uma perspectiva lúdica, profícua e integrada.

\section{Referências}

Aguiar, Ygor Q. de et al. (2015). Introdução à Robótica e Estímulo à Lógica de Programação no Ensino Básico Utilizando o Kit Educativo LEGO® Mindstorms. In: Anais dos Workshops do IV Congresso Brasileiro de Informática na Educação (CBIE 2015), p. 1418-1424.

Bacich, Lillian; Moran, José. "Metodologias Ativas para uma Educação Inovadora: Uma Abordagem Teórico-Prática. Porto Alegre: Penso Editora, 2017.

Bomfim, N.R. "A imagem da Geografia e do ensino da Geografia pelos professores das séries iniciais". Estudos Geográficos, Rio Claro, v.4, jun.2006, p.107-116.

De Oliveira, Gustavo B. et al. Kits Lego ${ }^{\circledR}$ Mindstorms ${ }^{\circledR}$ NXT como ferramenta de interação entre Engenharia e Ensino Médio. In: XLI COBENGE, 2013, Gramado - RS.

MARQUES, Antonio Manuel de Miranda. Utilização pedagógica de mapas mentais e de mapas conceptuais. 2008. 153 f. Dissertação (Mestrado em Expressão Gráfica, Cor e Imagem) Universidade Aberta de Portugal, Lisboa, 2008. 
Monteiro, S. B. S. et al. Uma Nova Abordagem de Ensino de Engenharia: Aprendizagem Baseada em Projetos (PjBL) na Disciplina PSP1 da Curso de Engenharia de Produção da UnB. In: XXXIX Congresso Brasileiro de Educação em Engenharia. ABENGE, 2011.

Puziski, Marcelo; Miglioranza, Silvia Maria Vanassi; Villas-Boas, Valquíria; Giovannini, Odilon. "Modelo Terra-Lua-Sol: Uma atividade interdisciplinar". Scientia Cum Industria, v.6, n.1, p.16-21, 2018.

Peralta, D.A., \& Guimarães, E.C. (2018). A robótica na escola como postura interdisciplinar: o futuro chegou para a Educação Básica?. Revista Brasileira de Informática na Educação - RBIE, 26 (1), 30-50. DOI: 10.5753/RBIE.2018.26.01.30.

SBC, Sociedade Brasileira De Computação (2017). "Referenciais de Formação em Computação: Educação Básica”. Disponível em: <http://bit.ly/2LMcevX>. Acesso em 17 out. 2018.

Savery, J. R. (2015). Overview of problem-based learning: Definitions and distinctions. Essential readings in problem-based learning: Exploring and extending the legacy of Howard S. Barrows, 9:5-15.

Sousa, Jessika Míirlla Farias de. Estratégias utilizadas nas aulas de geografia em turmas de nível médio a partir do subprojeto GEOGRAFIA/PIBID/UEPB. Trabalho de Conclusão de Curso (Graduação em Geografia), Universidade Estadual da Paraíba, Centro de Educação, 2017.

Vianna, M.; Vianna, Y.; Adler, I.K; Lucena, B; Russo, B. (2012). “Design Thinking: Inovação em Negócios". Rio de Janeiro: MJV Press. 